\title{
Anotaciones al componente pragmático en el Síndrome de Asperger (SA)
}

Notes on pragmatic component in Asperger Syndrome (AS)

\author{
Gonzalo Acosta P. \\ Pontificia Universidad Católica del Perú \\ g.acosta@pucp.pe
}

\section{RESUMEN}

En la presente nota se presenta un perfil de las principales habilidades pragmáticas dañadas en los individuos afectados por el Síndrome de Asperger (SA), entendiendo estas como aquellas que regulan el uso social del lenguaje, las cuales coordinan la atención a elementos extralingüísticos, propios de la interacción. En conciso, se describirán tres aspectos yuxtapuestos del uso lingüístico en la performance social: (a) los actos que expresan intenciones comunicativas, (b) las presuposiciones (c) y la organización del discurso social.

Palabras clave: Síndrome de Asperger (SA), Desarrollo Típico (DT), Autismo de Alto Rendimiento (HFA, siglas en inglés); Teoría de la mente.

\section{ABSTRACT}

In this article a profile of the main pragmatic skills damaged in individuals affected by Asperger's Syndrome (SA) is presented, understanding these as those that regulate the social use of language, which they coordinate attention to extra-linguistic elements, typical of interaction. In concise, three juxtaposed aspects of linguistic use in social performance will be described: (a) acts that express communicative intentions, (b) presuppositions (c) and the organization of social discourse.

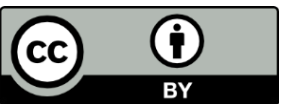


Keywords: Asperger syndrome, typical development, High Functioning $\mathrm{Au}-$ tism (HFA), Theory of mind.

El Síndrome de Asperger (en adelante, SA) es un trastorno generalizado del desarrollo sin afección fenotípica física, cuya etiología involucra factores genéticos (n. 1) ${ }^{1}$ (Briciet-Lauritsen et al. 2005) y pertenece al espectro autista, también vinculado frecuentemente, y a veces indiferenciado, del HFA (n. 2) (Frith 1991). Este síndrome revela dificultades en el reconocimiento de la información social y en el uso interaccional del lenguaje, mas un desarrollo lingüístico adecuado y hasta avanzado (n. 3), comportamiento repetitivo e intereses obsesivos de naturaleza idiosincrática y retraso en el desarrollo motor y la coordinación motriz (Martín-Borreguero 2004; Atwood 1998); además, los afectados no manifiestan retraso mental, sino un CI más bien en la media o hasta elevado (n. 4).

Con respecto a las características conductuales, en los SA resalta una inflexibilidad comportamental que se trasluce en una preocupación absorbente por un foco de interés o un número restringido de actividades, adhesión inflexible a rutinas y rituales estereotípicos y repetitivos, y una denotada preocupación por parte de los objetos (Atwood 1998).

El SA parece estar asociado a un perfil específico de habilidades cognitivas muy dispersas entre sí. Por un lado, es característico que el niño manifieste un avanzado desarrollo de las habilidades verbales que se observa en su actuación en las escalas verbales de los tests psicométricos, así como en las pruebas de memoria verbal y percepción auditiva (n. 5). De otro lado, el niño presenta una disfunción en el procesamiento de información viso-espacial que se manifiesta en su dificultad para la interpretación visomotora, percepción viso-espacial, memoria visual y desarrollo de conceptos no verbales.

Un aspecto diferenciador no marcado entre el autismo y el SA por el DSM -IV es la habilidad comunicativa deteriorada (n. 6), en menor medida en los SA (Dickerson et al. 2003), que se debe a la falta de algunas habilidades del uso pragmático (n. 7), que está severamente afectado en el caso de los autistas.

1 Hemos preferido colocar las notas al pie de este texto en un apartado hacia el final de este debido a su extensión 
Este aspecto del estudio del desarrollo cognitivo en los SA ha recibido bastante atención por parte de los investigadores.

Se ha afirmado que la disfunción pragmática del SA radica en su falta de empatía (n. 8, 9, 10). El concepto de teoría de la mente, definido como la capacidad de atribuir estados mentales a los otros, en este sentido, resulta iluminador, pues mediante la ausencia de él se explican las anormalidades específicas en el comportamiento social de los SA; las cuales afectan, particularmente, sus habilidades en el trato recíproco y la, ya mencionada, empatía (n. 11, 12) (Kennett 2002). La neurociencia ha identificado dos regiones cerebrales relacionadas a la mentalización dañadas en estos individuos (n. 13) (Pfeifer et al 1993). Martín-Borreguero advierte, sin embargo, que, en los SA, los resultados de la teoría de la mente deben interpretarse cuidadosamente; pese a todo, es la teoría que parece recoger mayor aceptación entre los investigadores y que pretende explicar conjuntamente SA y autismo (Frith 1991) (n. 14, 15, 16, 17).

El desarrollo pragmático refiere al aprender las reglas que entallan las formas del lenguaje (estructura lingüística) y el acto de expresar el significado para responder a las demandas sociales del contexto lingüístico y no lingüístico (n. 18). Ciertas habilidades pragmáticas en los SA se traslucen en dificultades comunicativas que provienen de una limitada capacidad para atender al desarrollo no verbal de las interacciones. Para notar la diferencia o el grado de diferencia entre un hablante con desarrollo típico (en adelante, DT) y un SA, aquí nos centraremos en tres dominios yuxtapuestos en el uso lingüístico: (a) los actos que expresan intenciones comunicativas, (b) las presuposiciones y (c) la organización del discurso social (Landa 2000; Martín-Borreguero 2005).

Los dominios (a), (b) y (c) presuponen la capacidad del uso de mecanismos atencionales para desentrañar las reglas sociales de comportamiento y cortesía; dicha capacidad es innata en los TD y supone en su funcionamiento la capacidad de considerar la perspectiva del otro; lo cual implica a su vez, la capacidad para hacer uso de una teoría de la mente: aspecto que parece ser adquirido, pero no innato en los SA (n. 19).

(a) La expresión de intenciones comunicativas refiere al uso directo o indirecto que tienen los hablantes para expresar un deseo o intención de acuerdo a la premisa de no daño a la 'imagen' del interlocutor (Koike y Memoria 2012). Es decir, de atentar contra la valía social que, tanto hablantes como oyentes, 
están de acuerdo en sostener durante el intercambio. Para el éxito social, es importante desarrollar habilidades que sirvan para reconocer situaciones en las que las intenciones pueden o deban ser expresadas indirectamente: aquí se hace necesario tener la flexibilidad lingüística apropiada para seleccionar formas no dañinas para expresar esta intención. Los SA muestran idiosincráticas formas de expresar dichas intenciones, una restringida variedad de intenciones expresadas y limitaciones en su habilidad por medir el grado de directividad en el que éstas son enunciadas, y así, causan la impresión de descortesía, rudeza o insensibilidad. La prosodia afectada en los SA también juega un papel importante en el reconocimiento de intenciones comunicativas (n. 20) (Tager-Flushberg 2003); a su vez, los SA tienen problemas para interpretar los significados intencionales de otros: muchas veces sus intenciones comunicativas suelen ser de naturaleza instrumental y dirigidas a satisfacer sus necesidades físicas y emocionales (Martín-Borreguero 2005), y toman las expresiones indirectas por su sentido literal.

(b) La presuposición refiere al conocimiento, expectativas y creencias que el hablante asume como compartidos con el interlocutor, lo cual requiere de una evaluación de la situación conversacional y el conocimiento atribuido (o presupuesto) al interlocutor, que incluye también la consideración extra discursiva a cualidades físicas (edad, género, etc.), estatus social del hablante, variables contextuales, historias de experiencias compartidas y contenido previo del discurso, para lo cual el hablante ajusta una serie de rasgos lingüísticos apropiados como la complejidad gramatical por usar, a partir de su repertorio comunicativo. Los SA tienen como rasgo característico la incapacidad de ajustar su producción lingüística, en consideración a las pistas contextuales cambiantes: aquí se notan el uso invariable de un estilo formal inapropiado. Se cree que su deficiente uso de las habilidades presuposicionales pueden deberse a su incomprensión de las pistas verbales y no verbales, así como su comprensión limitada de las pistas afectivas presentes en la entonación; además, su limitada comprensión de los significados implicados (implicancia) contribuye a un pobre reconocimiento de una solicitud de aclaración por parte del interlocutor, de allí que el SA tienda a los extensos monólogos.

(c) Nos referimos al discurso social como la estructuración de una serie de oraciones regidas por una cohesión temática y presuposiciones en el desarrollo 
de un tópico o varios temas. El mantenimiento de un tema es una habilidad compleja, que requiere la habilidad de usar flexiblemente usos gramaticales, entender y producir relaciones semánticas pertinentes, reconocer información compartida con los interlocutores y notar e interpretar el significado de marcas o pistas de cambio de tema, entre otras (n. 21). Un discurso coherente también depende de factores como una presentación bien organizada de la información y señales apropiadas que den a entender cómo los mensajes han de ser interpretados, esto implica, sobre todo, atender al desarrollo del discurso del hablante (Landa 2000).

Las bases del discurso como el prestar atención y la reciprocidad social son deficientes en el SA. La incapacidad de prestar atención está caracterizada por la ausencia o mínimo uso y/o comprensión del notar y mostrar gestos y los patrones de la mirada y estados emocionales cambiantes durante la interacción (Martín-Borreguero 2005). Para comprender actos comunicativos sociales, que son pobremente reconocidos -los cuales tendrán un impacto en habilidades como el establecimiento de la co-referencia (llevar el tema de la conversación)- es importante el uso del contacto visual para modular la longitud y cambio del turno, y destacar señales indicando el sentido no literal (Tager-Flushberg 2003); asimismo, las dificultades en el uso de señales sirven para mantener el tópico de conversación o el interés recíproco. Los SA tienden a ser percibidos como asociativos, ya que formulan cadenas de eslabones de interacción desconectados y descontextualizados (Martín-Borreguero 2005), lo que genera que cambien drásticamente los temas de conversación.

Así reunidas estas características se explican las diversas dificultades por las que atraviesan los SA en su trato diario con el mundo DT; en el que usualmente son percibidos como individuos anti-sociales e introspectivos. 


\section{Notas}

1. No se ha llegado a un consenso definitivo sobre la etiología del SA; sin embargo, como Briciet-Lauritsen et al, (2005) han investigado, existen pruebas suficientes para apoyar la comúnmente aceptada teoría de que existen factores genéticos involucrados en la etiología del autismo, SA y otros PPD's, aunque todavía con un estatus nosológico ambiguo.

2. Gillberg (1996) ha sugerido que, respecto a la etiología, el SA parece ser un trastorno predominantemente genético; y el HFA, un trastorno genético con un patrón de herencia diferente del SA o un trastorno causado por un daño cerebral. Por otro lado, Landa no diferencia HFA y SA respecto a su función pragmática. Frith (1991) anotaba que aún no es claro que se pueda trazar una línea divisora entre HFA y SA.

3. A decir verdad, pese a que el desarrollo lingüístico no sea una disfunción en los SA, se ha encontrado que, en una evaluación más profunda, algunos SA registran problemas en la combinación flexible de palabras, una gama restringida de significados y un déficit severo en la comprensión del lenguaje en contextos novedosos (Martin Borreguero 2004).

4. Estos rasgos son, en suma, las características identificadas por Asperger, las cuales han permanecido, en su mayor parte, inalterables hasta nuestros días; sin embargo, Asperger nunca llegó a definir criterios de diagnóstico específicos y cuantificables, lo que sucederá décadas después gracias al equipo de Cristopher Gillberg, cuyo aporte en la definición de pautas precisas de diagnóstico daría lugar a la primera definición operativa del SA (Martin Borreguero). Muchos, a su vez, han aportado y ampliado el criterio de diagnóstico del SA propuesto por Asperger, entre los cuales se cuentan a Lorna Wing, quien además familiarizó al mundo angloparlante con las ideas de Asperger en 1981 (Baron-Cohen 2010), Gillberg (1989-1991), Szatmari (1989), Tantam (1988-1991) y el ya conocido DSM-IV (1994) (Martin Borreguero 2004). 
5. A modo de síntesis, podemos decir que los SA manifiestan las siguientes características en relación a las habilidades lingüísticas: desarrollo adecuado de los componentes formales del lenguaje durante la etapa de la infancia temprana; ausencia aparente de retraso lingüístico; prosodia anómala y estilo conversacional egocéntrico, pedante y unilateral; gran facilidad para expresar ideas verbalmente; verbosidad marcada; vocabulario sofisticado e idiosincrático; el contenido de su conversación es de naturaleza compleja; referencias inusuales y ambiguas en la conversación con respecto a la información proporcionada por el interlocutor (Martin Borreguero 2004).

6. En su estudio, Miller \& Ozonoff (1997) reevaluaron clínicamente a los niños de las notas de Asperger bajo el criterio del DSM-IV, pero no encontraron una diferencia de diagnóstico entre estos y los autistas clásicos. El criterio de diagnóstico del DSM-IV no diferencia como SA a los sujetos descritos por Asperger, con lo que se sugiere que la verdadera naturaleza del SA no está totalmente captada por el criterio del DSM-IV.

7. Existe también ciertas hipótesis, todavía en debate, que plantean que el autismo y el SA comparten un continuum en su discapacidad social-comunicativa, lo cual indica que los SA serían el 'puente' entre el autismo y la normalidad (Baron-Cohen et al, 2001).

8. La empatía es la habilidad de atribuir estados mentales (Teoría de la Mente) a una persona/animal y se relaciona con la capacidad de dar una respuesta afectiva apropiada en base a la observación de los estados mentales del otro; esta articula componentes cognitivos y afectivos, lo cual nos permite entender las intenciones de otro, predecir su comportamiento y experimentar una emoción propiciada por las emociones de otro. Baron-Cohen et al (2004) reportó una baja puntuación en sus sujetos HFA y AS su test EQ, en el que se encontraron dificultades en la empatía. (Baron-Cohen et al, 2004).

9. En el DT, los niños de 3 años tienen muchos conocimientos sobre el funcionamiento psicológico de las personas: en esencia constituyen su visión de las personas como entidades que se relacionan con el mundo directamente tal y como es, de forma no representacional, y 
suelen fallar en las pruebas de falsas creencias (una prueba de la Teoría de la Mente), mientras que a los 4 o 5 años, los DT desarrollan un nivel representacional de las mentes, con la que comprenden que las entidades mentales son representaciones de la realidad, no la realidad misma (Villar 2001).

10. Baron-Cohen (2010) propone también la "teoría de la empatía-sistematización”, en la que la lectura de la mente no es más que el componente cognitivo de la empatía, mas no su elemento reactivo, el cual sería el elemento afectivo de la empatía.

11. Por otra parte, se sabe también que esta misma disfunción podría ser la causa de su incomprensión de estados mentales en los individuos autistas. A este respecto, la prueba de "Reading the Mind in the Eye” evalúa la Teoría de la Mente aplicada al reconocimiento de la atribución de un estado mental relevante a partir de la mirada (ejm: compasión): primer nivel de los test de teoría de la mente (y no la inferencia del contenido de dicho estado mental, segundo nivel de los test de teoría de la mente). Los HFA y SA evaluados demostraron dificultades para la lectura de la mente; cabe notar que esta evaluación fue llevada a cabo en adultos, sobre la base de una predicción de resultados en evaluaciones anteriores a niños autistas (Baron-Cohen, Wheelright et al, 2001).

12. Casi todas las habilidades pragmáticas necesitan de una teoría de la mente (Barbolla y García 1993).

13. Estudios neurocientíficos han llamado la atención sobre 4 regiones cerebrales clave implicadas en la mentalización y otros aspectos de la cognición normal social: temporal parietal juction (TPJ), MPFC dorsal (DMPFC), sulcus temporal posterior superior (Psts) y los polos temporales. Los procesos de atribución de perspectivas a un tercero, incluyendo el razonamiento acerca de las creencias y estados mentales, parecen ejecutarse en una región situada en la intersección entre el lóbulo parietal inferior y el gyrus temporal superior, también TPJ, zona afectada en los autistas (Pfeifer et al,1993).

14. La explicación de Teoría de la Mente ha sido cuestionada debido a la performance irregular de algunos SA en pruebas de Teoría de la 
Mente complejas: se pueden resolver las pruebas mediante el uso de la lógica (mecanismos compensatorios) (Landa 2000). En un estudio de evaluación de la teoría de la mente en autistas, Happé (1995) sugiere que el éxito de un grupo de autistas en dichas pruebas estaba estrechamente relacionado a una habilidad verbal superior (aspecto marcado en los autistas) cercana a la de los SA (competencia lingüística y comunicativa superior), con lo que sugiere que el éxito en las pruebas de teoría de la mente está estrechamente relacionado a un nivel verbal avanzado, como es el caso de los SA. Farrant et al (1999) observa algo parecido en relación al estado de la metamemoria en autistas.

15. Se puede superar el test de Sally-Ann, un test de la Teoría de la Mente, sin tener una comprensión intuitiva de los estados mentales. Parece que los SA pueden desarrollar una Teoría de la Mente alternativa, adquirida más tarde con considerables esfuerzos, la cual puede producir una base de creencias creciente y con potencial para mejorar con el tiempo, pero no permite la computación rápida y automática de la percepción social (Frith 1998). Hay estudios que indican que los SA pueden tener conocimientos acerca de la Teoría de la Mente de los otros, pero no son capaces de aplicar este conocimiento de forma eficaz, ya que parecen tener habilidades para intelectualizar los pensamientos o sentimientos del otro, pero tienen dificultades para usar estas habilidades cuando es necesario. A esto se le llama "carencia de control de coherencia central": la incapacidad para ver la importancia de los diferentes tipos de conocimientos y poder aplicarlos a un problema concreto (Atwood 1998).

16. El diferente proceso de mentalización en los autistas se ve confirmado en las pruebas efectivas mediante tomografía por emisión de protones, actualmente mediante resonancia magnética funcional (Cereols 2012).

17. La evaluación de la teoría de la relevancia permite hacer predicciones precisas acerca de los niveles de la competencia comunicativa que deberían corresponder a un primer o segundo orden de la habilidad de teoría de la mente (Happé 1993), predicciones que determinarían qué 
aspectos de la comunicación serán aprendidos y cuáles no. Esta teoría resulta la más adecuada para guiar la investigación de los problemas pragmáticos y su intervención temprana en los SA (Frith 1991).

18. La adquisición de reglas pragmáticas depende, en gran medida, de otros aspectos del desarrollo en otros subsistemas del lenguaje y sistemas cognitivos como la cognición y control ejecutivo de funciones, entre otros (Landa 2000).

19. Este aspecto resulta ser polémico entre los investigadores, cf. Cereols 2012.

20. La prosodia refiere a las propiedades de la señal discursiva usadas para modular y enfatizar el significado. Hay 3 niveles de la función prosódica: gramatical, pragmática y afectiva. El énfasis pragmático puede llamar la atención del hablante sobre nueva información o puede dibujar la atención del oyente sobre el significado del mensaje expresado, que puede no concordar con el significado literal. La prosodia afectiva atañe a los sentimientos y/o actitudes del hablante y puede incluir variaciones en el tono vocal y el rango del discurso. Se ha probado que los SA tienen dificultades no solo para reconocer y usar la prosodia afectiva (discurso monótono), sino también en la comprensión de la información prosódica expresada por otros; juntas dichas dificultades contribuyen significativamente a explicar la incapacidad de decodificar las intenciones comunicativas (Tager-Flushberg 2003). El cambio de tono, la inflexión o el énfasis en ciertas palabras puede alterar el significado de las enunciados u oraciones; los SA, al no poder reconocer los cambios de significado, tienen solo a rescatar el significado literal (Atwood 1998).

21. Los intercambios sociales en grupos implican una mentalización de segundo nivel y el reconocimiento de las relaciones de presuposición enlazadas en los hablantes colectivamente y en tiempo real: las relaciones de cada uno de ellos con todos los demás y cómo estas relaciones cambian la manera de actuar de cada uno de ellos. Los SA tienen grandes dificultades para la mentalización de grupos, en los que los intercambios aumentan de manera no lineal y el SA requiere tiempo 
48 Sorda \& Sonora (1) 2018

para aislar cada una de estos conocimientos y analizarlos con detenimiento (Cereols 2012). 


\section{Referencias bibliográficas}

Attwood, Tony. Asperger's syndrome: A guide for parents and professionals. Jessica Kingsley Publishers, 1998.

Borbolla, M., y Domingo García Villamisar. "La "teoría de la mente" y el autismo infantil: una revisión crítica," Revista complutense de educación, vol. 4, no. 2, 1993, pp. 11-28.

Baron-Cohen, Simon. Autismo y síndrome de Asperger. Alianza, 2010.---. Mindblindness: an essay on autism and theory of mind. MIT Press, 1995.

Baron-Cohen, Simon, et al. "The "Reading the Mind in the Eyes" Test revised version: a study with normal adults, and adults with Asperger syndrome or high-functioning autism." The Journal of Child Psychology and Psychiatry and Allied Disciplines, vol. 42, no. 2, 2001, pp. 241-251. https://doi.org/10.1111/1469-7610.00715

Baron-Cohen, Simon, et al. "The autism-spectrum quotient (AQ): Evidence from Asperger syndrome/high-functioning autism, malesand females, scientists and mathematicians." Journal of autism and developmental disorders, vol. 31 no. 1, 2001, pp. 5-17. https://doi.org/10.1023/A:1005653411471

Baron-Cohen, Simon, et al. "The Empathy Quotient: An Investigation of Adults with Asperger Syndrome or High Functioning Autism, and Normal Sex Differences." Journal of Autism and Developmental Disorders, vol. 34. no. 2, 2004, pp. 163-175. https://doi. org/10.1023/B:JADD.0oooo22607.19833.00

Cereols, Ramon. Descubrir el Asperger. Una amplia visión de este trastorno aún poco conocido escrita desde la experiencia personal. Promolibro, 2012.

Cobo, M. C., y E. Morán. El síndrome de Asperger: intervenciones psicoeducativas. Zaragoza: Asociación Asperger y TGD de Aragón, 2011

Dickerson, Susan, y Susan Calhoun. "Relationship between Asperger Syndrome and High-Functioning Autism." Learning and Behavior Problems in Asperger Syndrome, editado por Margot Prior. The Guilford Press, 2003.

Farrant, Annette, et al. "Metamemory in Children with Autism." Child Developmental, vol. 70, no. 1, 1999, pp. 107-131. https://doi.org/10.1111/1467-8624.00009

Frith, Uta. Asperger and his syndrome. Cambridge University Press, 1991.

---. "Autismo y Síndrome de Asperger," La esperanza no es un sueño, editado por Escuela Libre, 1998.

Hall, Kenneth. Soy un niño con síndrome de Asperger. Paidós, 2003.

Happé, Francesca. "Asperger's Syndrome." Theory of Mind and Communication in Autism editado por University of London, 1991. 
---. "Communicative competence and theory of mind in autism: A test of relevance theory." Cognition, vol. 48, 1993, pp. 101-119. https://doi.org/10.1016/0010-0277(93)90026-R

---. "The Role of Age and verbal Ability in the Theory of Mind Task Performance of Subjects with Autism." Child Development, vol. 66, no. 3, 1995, pp. 843-855. https:// doi.org/10.2307/1131954

Kennett, Jeanette. "Autism, Empathy and Moral Agency." The Philosophical Quarterly (1950-) ), vol. 52, no. 208, 2002, pp. 340-357. https://doi.org/10.1111/1467-9213.00272 Koike, D., y J. Memoria. "Análisis de la conversación." Fundamentos y modelos del estudio pragmático y socio pragmático del español editado por Susana de los Heros y Mercedes Niño-Murcia. Georgetown University Press, 2012, pp. 117-140.

Landa, Rebecca. "Social Language Use in Asperger Syndrome and High-Functioning Autism." Asperger Syndrome editado por Klin, Volkmar y Sparrow. The Guilford Press, 2000.

Lauritsen, Marlene, et al. "Effects of familial risk factors and place of birth on the risk of autism: a nationwide register-based study." Journal of Child Psychology and Psychiatry, vol. 46, no. 9, 2005, pp. 963-971. https://doi.org/10.1111/j.1469-7610.2004.00391.x Martín Borreguero, Pilar. El síndrome de Asperger: ¿excentricidad o discapacidad social? Alianza, 2004.

---. "Perfil lingüístico del individuo con síndrome de Asperger: implicaciones para la investigación y la práctica clínica." Revista de neurología, vol. 41, no. 1, 2005, pp. $115-122$.

Miller, Judith N., y Sally Ozonoff. "Did Asperger's cases have Asperger disorder? A research note." Journal of Child Psychology and Psychiatry, vol. 38, no. 2, 1997, pp. 247251. https://doi.org/10.1111/j.1469-7610.1997.tbo2354.x

Nordin, Viviann, y Christopher Gillberg. "Autism spectrum disorders in children with physical or mental disability or both. I: Clinical and epidemiological aspects." Developmental Medicine \& Child Neurology, vol. 38, no. 4, 1996, pp. 297-313. https://doi.org/10.1111/j.1469-8749.1996.tb12096.x

Parish-Morris, J. et al. "Children with Autism Illuminate the Role of Social Intention in Word Learning." Child Development, vol. 78, no. 4, 2007, pp. 1265-1287. https://doi. org/10.1111/j.1467-8624.2007.01065.x

Pfeifer, J.H et al. "Neural Correlates of Direct and Reflected Self-Appraisals in Adolescents and Adults: When Social Perspective-Taking Informs Self-Perception." Child 
Development, vol. 80, no. 4, 2009, pp. 1016-1038. https://doi.org/10.1111/j.14678624.2009.01314.x

Swensen, L. et al. "Processes of Language Acquisition in Children with Autism: Evidence from Preferential Looking." Child Development, vol.78, no.2, 2007, pp. 542-557. https://doi.org/10.1111/j.1467-8624.2007.01022.x

Tager-Flushberg, Helen. "Effects of Language and Communicative Deficits on Learning and Behavior." Learning and Behavior Problems in Asperger Syndrome editado por Margot Prior. The Guilford Press, 2003.

Villar, Feliciano. "Nuevas Perspectivas individuales en el desarrollo". Psicología Evolutiva y Psicología de la Educación, 2001.

Zardaín, Pilar y Gema Trelles García. El síndrome de Asperger. Asociación Asperger de Asturias, 2009 